\title{
Domain-walls formation in binary nanoscopic finite systems
}

\author{
A. Patrykiejew, S. Sokołowski \\ Department for the Modelling of Physico-Chemical Processes, Faculty of Chemistry, \\ Maria Curie-Sklodowska University, 20031 Lublin, Poland
}

Received February 24, 2014, in final form May 6, 2014

\begin{abstract}
Using a simple one-dimensional Frenkel-Kontorowa type model, we have demonstrated that finite commensurate chains may undergo the commensurate-incommensurate (C-IC) transition when the chain is contaminated by isolated impurities attached to the chain ends. Monte Carlo (MC) simulation has shown that the same phenomenon appears in two-dimensional systems with impurities located at the peripheries of finite commensurate clusters.
\end{abstract}

Key words: binary mixture, commensurate-incommensurate transitions, Monte Carlo simulation, finite systems, Frenkel-Kontorova model

PACS: $64.70 . R h, 68.55 . L n, 64.60 . a n$

\section{Introduction}

In modern nanotechnologies one often deals with very small systems of countable numbers of atoms or molecules. In such cases, the finite size and boundary effects are large and bound to significantly affect the properties of the system with respect to its bulk counterpart [1]. Another important problem is the purity of small systems. While tiny amounts of impurities may be unimportant in macro-scale, the behavior of nanoscopic systems is considerably influenced even by a small number of impurity atoms [2]. Among the systems in which the presence of impurities may be of importance are those exhibiting the C-IC transition.

The C-IC transitions have been experimentally observed in a variety of systems including adsorbed films, [3-6] intercalated compounds [7] 8] composite crystals [9] and magnetically ordered structures of rare-earth compounds [10]. Theoretical studies of C-IC transitions have focused on the domain wall description of incommensurate phases [11-14]. According to the domain wall formalism, the IC phase is a collection of $\mathrm{C}$ domains separated by domain walls. The density within the domain walls may be lower or higher than the density of commensurate domains. In the former case, the walls are light and superlight, while in the latter the walls are heavy and superheavy [13].

The simplest theoretical approach which predicts the formation of domain walls is the one-dimensional Frenkel-Kontorova model [15]. The original FK model assumes that an infinite chain of atoms interacting via harmonic potential at zero temperature is subjected to a periodic (sinusoidal) external field. Depending on the misfit between the equilibrium distance of the harmonic potential, the period and amplitude of the external field, the FK model is capable of describing the C-IC transition. The FK model has been extended to two-dimensional systems [16, 17] to mixtures [18], systems with disorder [19] and has also been used to study finite chains [20-22].

The NPT Monte Carlo simulation has demonstrated [23, 24] that finite one-dimensional chains, either uniform or subjected to periodic field, exhibit structures that cannot appear in infinite chains. In particular, it has been shown that the chain experiences very large density fluctuations. In the case of chains on a periodic substrate, a number of different structures (registered, free floating, domain-wall 
incommensurate and resulting from the chain fragmentation) have been found to appear during a single run.

In one of our recent papers [25], we have shown that finite two-dimensional clusters of $\mathrm{Kr}$ adsorbed on graphite undergo the C-IC phase transition when contaminated by small amounts of Ar atoms. Computer simulation has demonstrated that the transition occurs already when the boundaries of a finite krypton island are covered with a single layer of argon atoms.

In this paper we address the issue of the influence of impurities on the behavior of finite one- and twodimensional (1D and 2D) systems. We are interested in the effects of impurities located at the peripheries of finite 1D chains and 2D clusters of atoms subjected to the periodic external field. The field is assumed to be strong enough to enforce the formation of commensurate structures in pure systems and we consider the possibility of the C-IC transition driven by the presence of impurities. The paper is organized as follows. In the next section we discuss the behavior of one-dimensional systems in the framework of a modified Frenkel-Kontorova model. Then, in the third section we consider two-dimensional finite systems studied by Monte Carlo simulation. In the final section we summarize our findings.

\section{One-dimensional Frenkel-Kontorova model}

At first, we have considered a simple 1D finite chain of atoms at zero temperature and used the Frenkel-Kontorova (FK) model [15]. The energy of a finite chain consisting of $N$ atoms subjected to a periodic external potential and containing impurities can be written as follows:

$$
E=\frac{1}{2}\left\{\sum_{i=1}^{N-1} K_{i}\left[x_{i+1}-x_{i}-b_{i}\right]^{2}+\sum_{i=1}^{N} v_{i}\left[1-\cos \left(2 \pi x_{i} / a\right)\right]\right\},
$$

where $K_{i}$ and $b_{i}$ are the elastic constant and the equilibrium distance for the pair $(i, i+1)$ and $v_{i}$ is the amplitude of the external field for the $i$-th particle. Having introduced the displacements $u_{i}=x_{i} / a-p i$ $(i=1,2, \ldots N)$ with $p$ being a positive integer (in this work we set $p=2$ ), the energy given by eqn.(2.1) can be rewritten in units of $K_{0} a^{2} / 2$ ( $K_{0}$ being the elastic constant for a pure chain) as follows:

$$
E=\sum_{i=1}^{N-1} \hat{K}_{i}\left[u_{i+1}-u_{i}-m_{i}\right]^{2}+\sum_{i=1}^{N} \hat{v}_{i}\left[1-\cos \left(2 \pi u_{i}\right)\right]
$$

In general, the elastic constants $\hat{K}_{i}$ (the misfits $m_{i}$ ) can assume one of three possible values $k_{0,0} \equiv 1$, $k_{0, \mathrm{im}}$ or $k_{\mathrm{im}, \mathrm{im}}\left(m_{0,0}, m_{0, \mathrm{im}}\right.$ or $\left.m_{\mathrm{im}, \mathrm{im}}\right)$ depending on the composition of the pair $(i, i+1)$, and the amplitude $\hat{v}_{i}=v_{i} / a^{2} K_{0}$ is equal either to $v_{0}$ or to $v_{\text {im. }}$.

In order to find the equilibrium configuration of the chain, the energy should attain its minimum value, specified by the condition stating that the forces $f_{i}=-\partial E / \partial u_{i}=0$ for all $i$. We consider the systems in which a single impurity atom is located at one end of the chain (class I) and the systems with two impurity atoms located at both ends of the chain (class II) and put $k_{0, \mathrm{im}}=k_{\mathrm{im}}$ and $m_{0, \mathrm{im}}=m_{\mathrm{im}}$. The behavior of pure chains depends on $m_{0}$ and $\nu_{0}$. For the assumed value of $m_{0}=-0.1$, pure chains are commensurate when $v_{0}$ exceeds the critical value $v_{0, \mathrm{c}} \approx 0.004950$. The calculations have been done for $N$ between 21 and 401 and $\nu_{0}=0.006$.

The first series of calculations have been carried out for $k_{\mathrm{im}}=1.0$, while $v_{\mathrm{im}}$ and $m_{\mathrm{im}}$ have been varied. Figure 1 shows the example of the results obtained for the chains with $N=41, m_{\mathrm{im}}=-0.25$ and different values of $v_{\mathrm{im}}$. The lower and upper panels of figure 1 show the results for the systems of class I and II, respectively. It is evident that the systems belonging to both classes exhibit a qualitatively similar behavior. Of course, the systems of the class I lack the symmetry of atomic displacements with respect to the central atom. For $v_{\text {im }}$ lower (higher) than $v_{\mathrm{im}, \mathrm{l}}\left(\nu_{\mathrm{im}, \mathrm{u}}\right)$, the chain remains commensurate, while for intermediate values of $v_{\text {im }}$, there appear incommensurate structures with domain walls. When $v_{\text {im }}$ is lower than $v_{\mathrm{im}, 1}$, the energy cost to put the impurity out of registry position is low and the impurity can exhibit large displacements from the commensurate position, while the rest of the chain assumes a commensurate structure due to the domination of the surface over the elastic interaction. On the other hand, when $v_{\mathrm{im}}>v_{\mathrm{im}, \mathrm{u}}$, the impurity is strongly pinned by the surface potential and the chain retains 


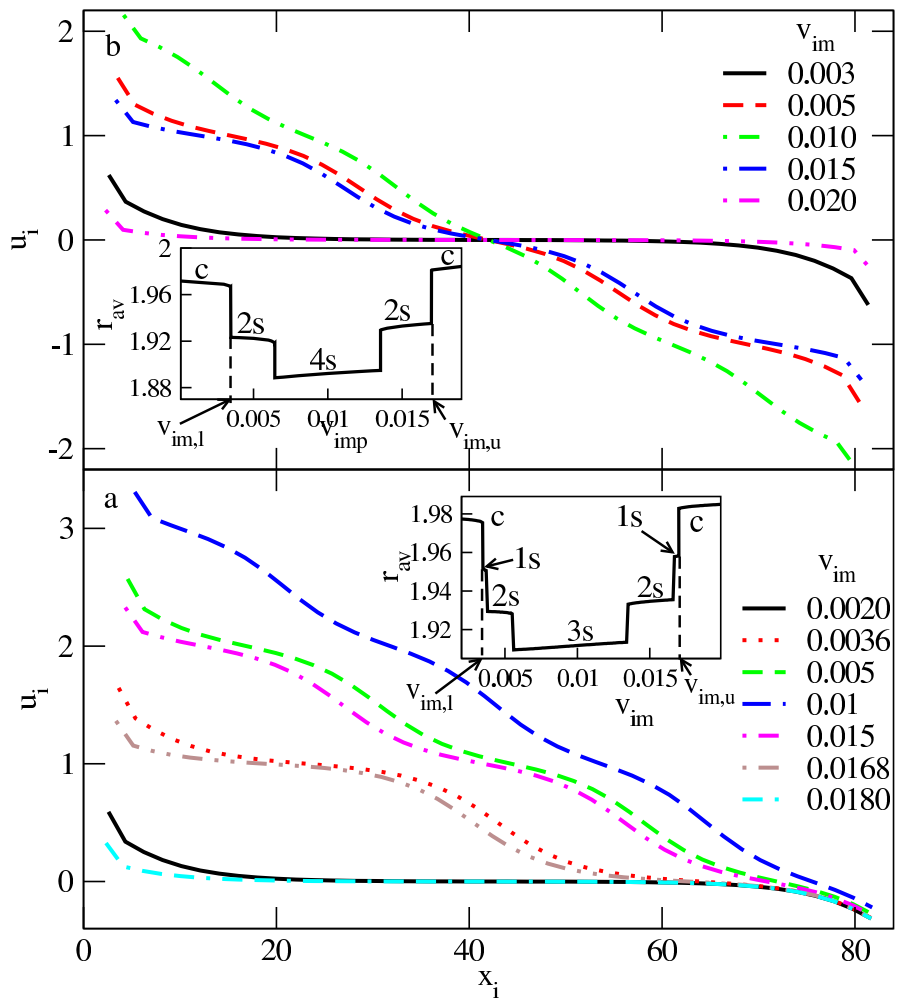

Figure 1. (Color online) Atomic displacements vs. atomic positions for the systems of class I (part a) and II (part b) and different values of $v_{\text {im }}$ (given in the figure). The calculations have been done $N=41$, $m_{\mathrm{im}}=-0.25$ and $k_{\mathrm{im}}=1.0$. The insets show the changes of the average nearest-neighbor distance vs. $v_{\text {im }}$ The regions marked by $C$ and $k s(k=1,2,3,4)$ correspond to the commensurate structure to the incommensurate structures with $k$ domain walls, respectively.

the commensurate structure. Figure 1 shows that when $v_{\mathrm{im}}>v_{\mathrm{im}, \mathrm{u}}$, the displacements of impurities are considerably lower than when $v_{\text {im }}<v_{\text {im }, 1}$.

For intermediate values of $v_{\text {im }}$, the gain in elastic energy due to transition into the incommensurate structure is larger than the loss of surface energy. The insets to figure 1 show the changes of the average nearest-neighbor distance with $\nu_{\mathrm{im}}$. In both classes of systems, we have found a series of transitions characterized by a different number of domain walls. The calculations for chains with $N$ up to 401 have shown that in longer chains a larger number of structures appear, and it seems that in the limit of $N \rightarrow \infty$ the transitions form a harmless staircase [11].

The values of $v_{\mathrm{im}, \mathrm{l}}$ and $v_{\mathrm{im}, \mathrm{u}}$ change with $m_{\mathrm{im}}$ and there is a critical value of $m_{\mathrm{im}, \mathrm{c}}$ for which the difference $\Delta v_{\mathrm{im}}=v_{\mathrm{im}, \mathrm{u}}-v_{\mathrm{im}, \mathrm{l}}$ goes to zero (figure 2). In the particular examples considered here $(N=41$ and $\left.k_{\mathrm{im}}=1.0\right), m_{\mathrm{im}, \mathrm{c}} \approx-0.1856$ for the systems of class I and II. The inset to figure 2 shows that $\Delta v_{\mathrm{im}}$ scales with $m_{\mathrm{im}, \mathrm{c}}-m_{\mathrm{im}}$ as follows:

$$
\Delta \nu_{\mathrm{im}} \propto\left(m_{\mathrm{im}, \mathrm{c}}-m_{\mathrm{im}}\right)^{1 / 2}, \quad k=1,2 .
$$

The same scaling appears for the transitions between different incommensurate structures, but the value of $m_{\mathrm{im}, \mathrm{c}}$ for each transition is different (figure 2).

We have then investigated the effects due to changes in the magnitude of $k_{\mathrm{im}}$. Figure 3 gives the example of results for the systems of class I. We see that the values of $v_{\mathrm{im}}$ at the transitions $C-1 s, 1 s-2 s$ and $2 s-3 s$ are nearly independent of $k_{\text {im }}$, while those corresponding to transitions $3 s-2 s, 2 s-1 s$ and $1 s-C$ (leading to the recovery of commensurability), exhibit a logarithmic dependence on $k_{\text {im }}$.

The logarithmic dependence of $v_{\mathrm{im}}$ on $k_{\mathrm{im}}$ at transitions $3 s-2 s, 2 s-1 s$ and $1 s-C$ has the same origin as the C-IC transition in a pure FK model [11]. On the other hand, the mechanism of transitions leading to commensurability when $v_{\text {im }}$ becomes very low is different. For sufficiently low $v_{\text {im }}$, the energy cost to put 


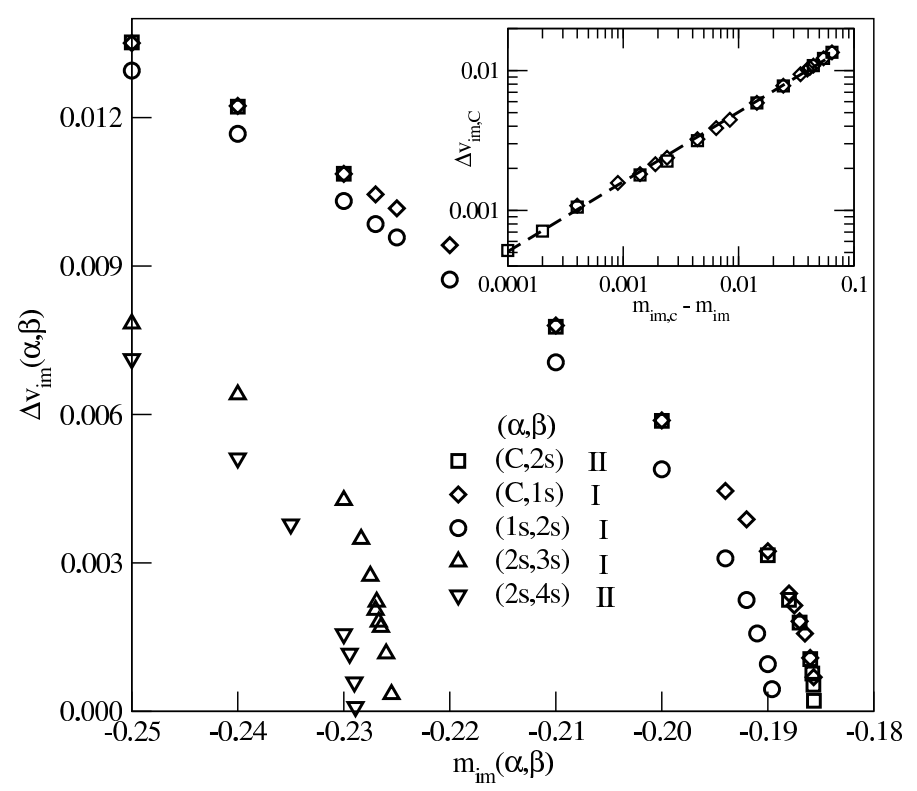

Figure 2. The phase diagrams showing the dependence of the difference between the upper and lower values of $\nu_{\mathrm{im}}$ at the transition points $\left(\Delta \nu_{\mathrm{im}}(\alpha, \beta)\right)$ between different structures $(\alpha, \beta)$ and the impurity misfit $m_{\mathrm{im}}(\alpha, \beta)$. The calculations have been done for $N=41$ and $k_{\mathrm{im}}=1.0$ and for the classes I and II. The inset shows the scaling plot for the C-IC transition.

the impurity out of registry position and to restore the commensurate positions in the rest of the chain is low. Consequently, the impurity can exhibit a large displacement from the commensurate position, while the rest of the chain assumes a commensurate structure due to the domination of surface energy over the elastic energy. This is illustrated in the inset to figure 3 , which shows atomic displacements for the

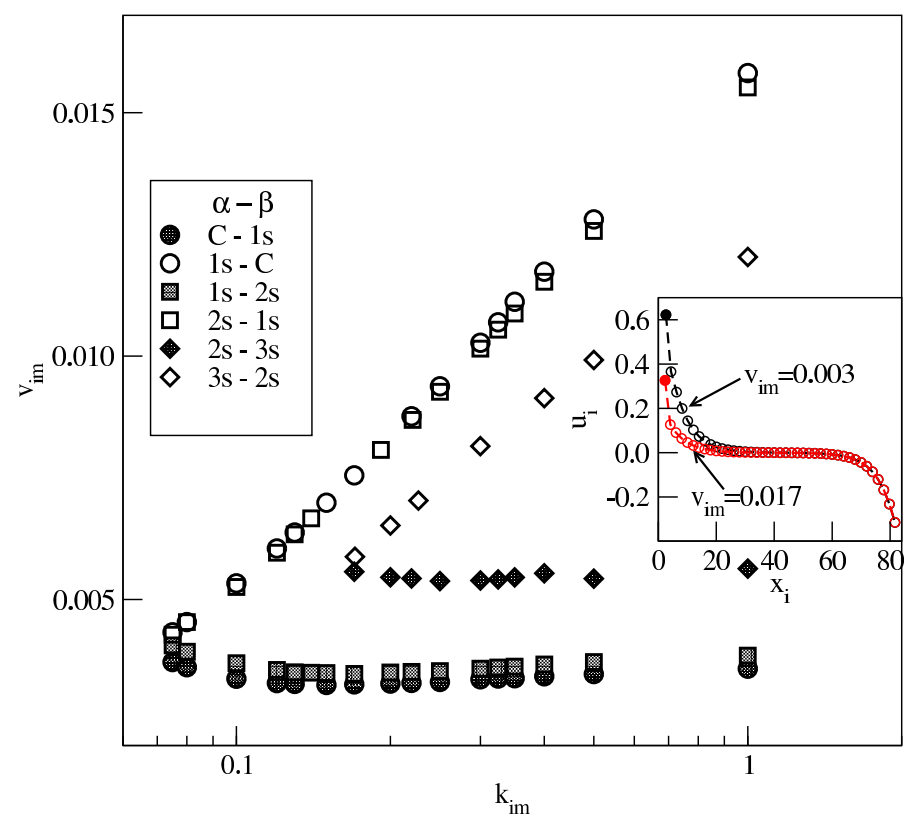

Figure 3. (Color online) The phase diagrams showing the dependence of $v_{\text {im }}$ on $k_{\text {im }}$ at the transition points between different structures. The calculations have been done for $N=41$ and $m_{\mathrm{im}}=-0.24$. The inset shows the atomic displacements versus actual atomic positions in the commensurate phase for $v_{\text {im }}=0.003$ and $v_{\text {im }}=0.017$ when $k_{\text {im }}=1.0$. Filled symbols mark the impurity atom. 
systems with $k_{\mathrm{im}}=1.0$ and $v_{\mathrm{im}}=0.003$ and $v_{\mathrm{im}}=0.017$, while the rest of the parameters have been kept the same as in the main figure. Of course, there is an asymmetry of displacements in the chain but it is very small. In the commensurate phase at high values of $v_{\mathrm{im}}=0.017$, the displacements at both ends are nearly the same.

Another question is whether a single impurity can drive the incommensurate system into commensurability? The answer is no. On the other hand, two impurities located at both ends of the chains do lead to the recovery of commensurability when $v_{\mathrm{im}}$ is sufficiently high. This is just the same as with the rope pinned either to one or two walls. In the first case, the rope hangs down freely. In the latter, the rope pinned to the opposite walls can be expanded to some extent. In the harmonic approximation, the chain always retains integrity, although when the interaction potential allows for dissociation, the chain may rupture 23 24, 26] rather than restore the C structure.

\section{Two-dimensional finite clusters}

The phenomenon of reentrant commensurability is not restricted to the above discussed simple 1D model. It also appears in more realistic 2D systems in the ground state and at finite temperatures. To demonstrate this, we have performed MC simulation in the canonical ensemble for finite clusters decorated with impurities at the boundaries. The particles of $A$ and $B$ (impurity) have been placed in the substrate field

$$
v_{i}(x, y)=-V_{i}\left\{\cos \left(\mathbf{q}_{1} \mathbf{r}\right)+\cos \left(\mathbf{q}_{2} \mathbf{r}\right)+\cos \left[\left(\mathbf{q}_{1}-\mathbf{q}_{2}\right) \mathbf{r}\right]\right\}
$$

corresponding to the graphite basal plane, where $V_{i}$ is the amplitude of the potential for the $i$ th component and $\mathbf{q}_{1}$ and $\mathbf{q}_{2}$ are the reciprocal lattice vectors of the graphite basal plane. The initial configurations have been a hexagon of a perfectly ordered $(\sqrt{3} \times \sqrt{3}) R 30^{\circ}$ structure consisting of $N_{A} z$ atoms of $A$ and $N_{B}$ atoms of $B$ placed along the one edge (case I), the adjacent three edges (case II) or along all six edges (case III) of the cluster. The interaction between the particles has been represented by the LJ $(12,6)$ potential with the fixed $\sigma_{A A}=1.46 a(a=2.46 \AA$ is the graphite lattice constant taken as the unit of length) and $\varepsilon_{A A} / k_{\mathrm{B}}=170 \mathrm{~K}$ (assumed to be a unit of energy) and $V_{A}^{*}=V_{A} / \varepsilon_{A A}=0.12$. In order to reduce the tendency of $B$ atoms towards aggregation, we have put $\varepsilon_{B B}=0.5 \varepsilon_{A A}$, while $\varepsilon_{A B}$ has been assumed to be equal to $\varepsilon_{A A}$. The parameters $\sigma_{B B}=\sigma_{A B}$ and $V_{B}$ have been varied.

The simulations have been carried out for the systems with $N_{A}=271$ and $N_{B}=11$ (case I), $N_{B}=33$ (case II) and $N_{B}=66$ (case III). Besides, we have also performed some runs for a larger system corresponding to case III, with $N_{A}=811$ and $N_{B}=114$ as well as with $N_{A}=1189$ and $N_{B}=138$. The simulation has been performed at reduced temperatures $T^{*}=k_{\mathrm{B}} T / \varepsilon_{A A}$ between 0.005 and 0.3 . The formation of domain walls in the system has been monitored using the order parameter [25, 27]

$$
\phi(\mathbf{r})=\cos \left(\mathbf{q}_{1} \mathbf{r}\right)+\cos \left(\mathbf{q}_{2} \mathbf{r}\right)+\cos \left[\left(\mathbf{q}_{1}-\mathbf{q}_{2}\right) \mathbf{r}\right]
$$

and assuming that the atom is commensurate (incommensurate) when $\phi>0(\phi \leqslant 0)$. We have calculated the average numbers of incommensurate atoms $A$ and $B$ neglecting the atoms located at the patch boundaries, i.e., those with less than 5 nearest neighbors.

It has been found that for any value of $\sigma_{B B}^{*}=\sigma_{B B} / a$ between 1.34 and 1.37 and for sufficiently low and sufficiently high values of $V_{B}^{*}$, the systems remain commensurate, while for the intermediate values of $V_{B}^{*}$, there appear networks of heavy walls separating commensurate domains.

In general, the results at low temperatures are qualitatively very similar to those obtained for 1D FK model. In particular, we have observed the formation of IC structures in the cases I, II and III, though the structure of the domain-wall networks is different in all cases. In general, the walls tend to assume the orientations perpendicular to the edges decorated with the impurity atoms. Therefore, in case III, we have found regular networks like that given in the leftmost part of figure 4 . On the other hand, the systems with only one or three adjacent edges decorated by impurities form irregular networks of domain walls (see the middle and rightmost panels to figure 4).

The region of $V_{B}^{*}$ over which the IC phase is stable at low temperatures gradually decreases when the size of impurity atoms increases, so that for sufficiently large impurity atoms, only the $C$ phase occurs independently of the magnitude of $V_{B}^{*}$ (see figure 5). The calculations for the larger system with $N_{A}=811$ 


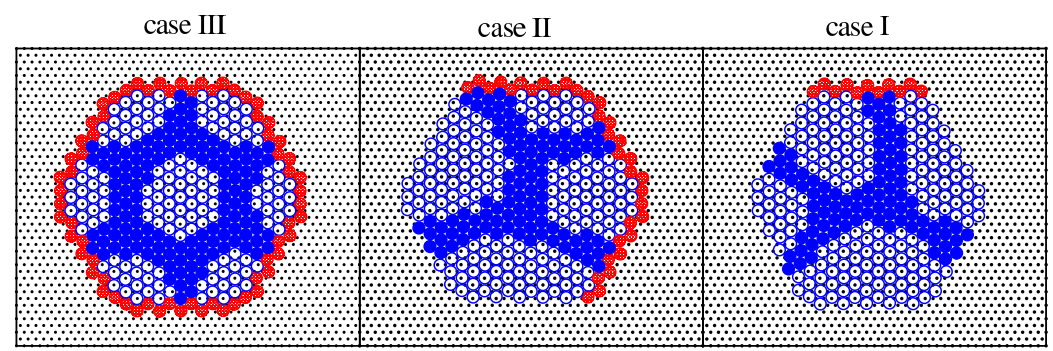

Figure 4. (Color online) The examples of snapshots for the system with $\sigma_{B B}^{*}=1.36$ and $V_{B}^{*}=0.12$ at $T^{*}=0.02$ corresponding to the three cases considered. Shaded circles represent $B$ atoms. Open (filled) circles correspond to the commensurate (incommensurate) $A$ atoms.

and $N_{B}=114$ (case III) have shown that maximum value of $\sigma_{A B}^{*}$ above which the C-IC transition does not appear slightly increases with the system size. Moreover, the simulations performed for larger systems have also demonstrated that at low temperatures the size of $\mathrm{C}$ domains does not change and only their number increases. This result is quite similar to that obtained for 1D FK model for the chains of different length. This is demonstrated by the snapshots given in the leftmost part of figure 4 and in figure 6 .

The impurity induced changes of the surface stress are responsible for the C-IC transition, which leads to the compression of the finite island. When the amplitude of the impurity surface potential, $V_{B}^{*}$, is very low, the presence of impurities at the cluster boundaries does not appreciably affect the strain of the atoms in the cluster. Therefore, the surface stress of the impurity decorated island does not match differently from that of the pure system. For sufficiently large values of $V_{B}^{*}$, the impurities are strongly pinned over the surface potential minima as well as do not enlarge the strain in the island. Therefore, for sufficiently low and high $V_{B}^{*}$, the system retains the $C$ structure. For intermediate values of $V_{B}^{*}$, the competing atom-atom and atom-external field interactions lead to a sufficiently large increase of a surface stress in order to trigger the C-IC transition. One should note a similarity of the herein reported impurity driven C-IC transition to the behavior of metal nanowires [28] and to the formation of IC phases in the subjected to uniaxial compression self-assembled monolayer gold nanoparticles supported on a fluid [29].

The results presented here describe only the low temperature behavior of the systems. The simulation has demonstrated that upon an increase of temperature, the IC structure gradually changes and the C structure is restored at temperatures still below the melting point of the cluster. The effects of thermal excitations on the domain-wall networks will be discussed elsewhere.

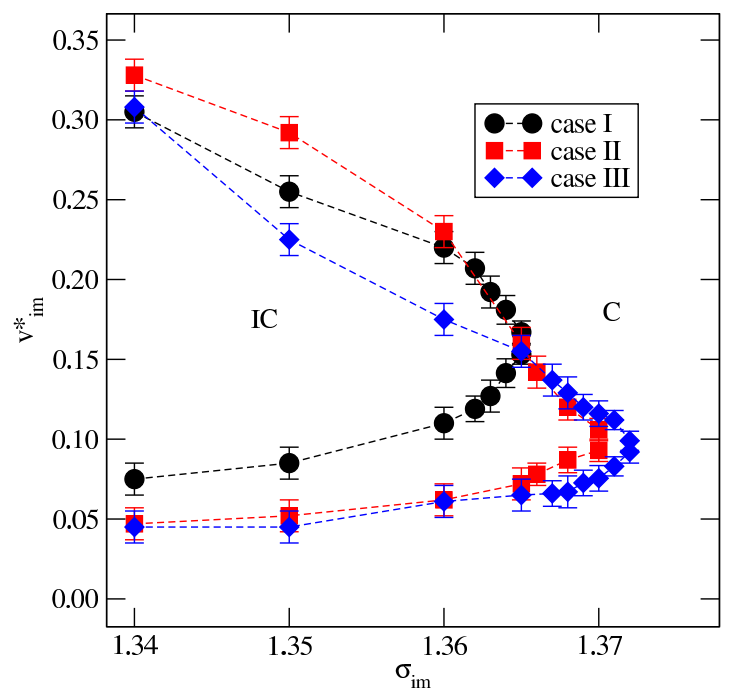

Figure 5. (Color online) The phase diagram showing the locations ( $V_{B}^{*}$ versus $\sigma_{A B}^{*}$ ) of C-IC transition in the limit of $T^{*} \rightarrow 0$ in the systems with $N_{A}=271$ and different concentration of impurity atoms. 


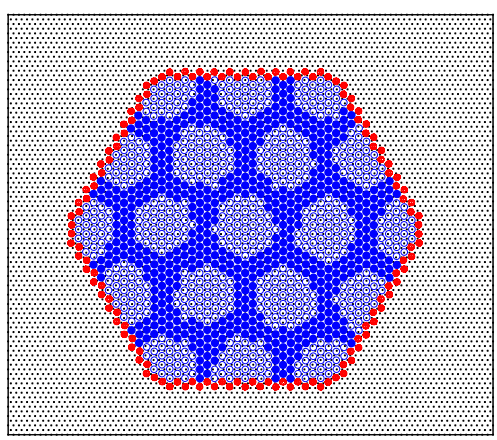

Figure 6. (Color online) The example of snapshot for the system with $N_{A}=1189$ and $N_{B}=138, \sigma_{A B}=$ 1.365 and $V_{B}^{*}=0.08$ recorded at $T^{*}=0.01 \mathrm{~K}$. Shaded circles represent $B$ atoms. Open and filled circles correspond to the commensurate and incommensurate $A$ atoms.

\section{Summary}

In this work we have studied the impurity driven commensurate-incommensurate transitions in oneand two-dimensional finite systems at zero temperature. In the case of one-dimensional finite chains, we have considered the situations in which the impurity is located at one and two ends of the chain. It has been shown that in both situations, the C-IC transition occurs when the amplitude of the external field experienced by the impurity atoms falls into the region between the lower and upper threshold values. These limiting values of $v_{\text {im }}$ depend upon the parameters characterizing the interaction between the atoms in the main chain, the amplitude of external field acting on the main chain atoms and the interaction between the main chain and the impurities. The number of solitons (domain walls) in the IC structure is different for the chains with one and two ends decorated with the impurity atoms and also depends upon the $v_{\mathrm{im}}$. This behavior is a consequence of different symmetry properties of the system.

In the case of two-dimensional finite clusters, a very similar C-IC transition has been found. In particular, we have observed that the transition occurs when only a part of the cluster boundary is covered with a single row of impurity atoms. Also, by analogy to the results of one-dimensional FK model, the transition occurs only between the lower and upper threshold values of $v_{\text {im }}$.

It should be noted that the C-IC transition observed in two-dimensional systems occurs only when the parameters entering the potential describing the interactions between $A A, A B$, and $B B$ atoms are suitably chosen. First of all, the components $A$ and $B$ should not exhibit the tendency towards mixing. Otherwise, the impurity atoms $B$ are likely to penetrate the patch and different scenarios are possible. One, is the formation of a mixed commensurate phase. This has been found in the case of Ar-Kr finite patches at sufficiently high temperatures [25], when the $A B$ interaction potential parameters were obtained using the standard Lorentz-Berthelot mixing rules. The same mixture exhibits a different behavior at low temperatures, and does undergo the C-IC transition, although with the domain walls preferentially made of $\mathrm{Ar}$ atoms. Another requirement is that atoms $A$ should tend to order into the $C$ phase, while atoms $B$ should order into the IC phase. This imposes certain restrictions on the choice of $\sigma_{A A}^{*}, \sigma_{B B}$ and the values of $V_{A}^{*}$ and $V_{B}^{*}$. In particular, $\sigma_{A A}^{*}$ and $V_{A}^{*}$ should assume the values ensuring that a pure $A$ patch is commensurate, but are likely to undergo the C-IC transition when the density exceeds the monolayer capacity or when subjected to an external force due to the presence of impurity atoms along the patch boundaries. Thus, $V_{A}^{*}$ cannot be too low or to high and the misfit between the sizes of surface lattice and adsorbate atoms is rather small. A good example of such a system is krypton adsorbed on graphite [5]. In fact, the parameters $\sigma_{A A}^{*}$ and $V_{A}^{*}$ used here are rather close to those describing the krypton adsorbed on the graphite basal plane [25]. On the other hand, the values of $\sigma_{B B}$ and $V_{B}^{*}$ should favor the formation of IC phase by pure component $B$. Thus, the misfit between the size of $B$ atoms and the surface lattice 
should be sufficiently large (it can be positive as well as negative) and $V_{B}^{*}$ should be sufficiently small. However, there is still another requirement for the appearance of C-IC transition in finite patches. The $A B$ interaction should be sufficiently strong so that the layer of $B$ atoms along the patch boundary is stable. Otherwise, the atoms $B$ would prefer to form clusters rather than stay at the patch boundary. Besides, this condition is also necessary to exert a sufficiently large force upon the atoms $A$ inside the finite patch to trigger the C-IC transition. Usually, the C-IC transition occurs only when the film density exceeds the density of a fully filled commensurate phase. For the C-IC transition to occur in finite patches, the stability of the $C$ phase should be low enough so that a rather small force exerted by a thin layer of impurity atoms along the patch boundary is able to drive the C-IC transition. From our earlier studies of adsorption of $\mathrm{Ar}-\mathrm{Kr}, \mathrm{Ar}-\mathrm{Xe}$ and $\mathrm{Kr}$-Xe mixtures [25, 30,32] on graphite it follows that only in the case of Ar-Kr mixture the C-IC transition occurs at submonolayer densities in finite patches of adsorbed phase.

One expects that at higher temperatures, thermal excitations may considerably influence the effects observed. This problem is currently under study and the results will be published elsewhere.

\section{Acknowledgements}

This work was supported by ERA under the Grant PIRSES 268498.

\section{References}

1. Esfarjani K., Mansoori G.A., In: Handbook of Theoretical and Computational Nanotechnology, Vol. 10, Rieth M., Schommers W. (Eds.), American Scientific Publishers, Los Angeles, 2005, 1-45.

2. Hwang I.-S., Fang C.-K., Chang S.-H., Phys. Rev. B, 2011, 83, 134119. doi 10.1103/PhysRevB.83.134119

3. Jaubert M., Glachant A., Bienfait M., Boato G., Phys. Rev. Lett., 1981, 46, 1679; doi 10.1103/PhysRevLett.46.1679

4. Krim J., Suzanne J., Shechter H., Wang R., Taub H., Surface Sci., 1985, 162, 446; doi 10.1016/0039-6028(85)90933-1.

5. Stephens P.W., Heiney P.A., Birgeneau R.J., Horn P.M., Moncton D.E., Brown G.S., Phys. Rev. B, 1984, 29, 3512; doi $10.1103 /$ PhysRevB.29.3512

6. Usachov D., Dobrotvorskii A.M., Varykhalov A., Rader O., Phys. Rev. B, 2008, 78, 085403; doi 10.1103/PhysRevB.78.085403

7. Clarke R., In: Ordering in Two Dimensions, Sinha S.K. (Ed.), North-Holland, Amsterdam, 1980, p. 53-58.

8. Li L.J., Lu W.J., Zhu X.D., Ling L.S., Qu Z., Sun Y.P., Europhys. Lett., 2012, 97, 67005; doi $10.1209 / 0295-5075 / 97 / 67005$

9. Nuss J., Pfeiffer S., van Smallen S., Jansen M., Acta Crystalogr. B, 2010, 66, 27; doi 10.1107/S0108768109053312

10. Vokhmyanin A.P., Lee S., Jang K.-H., Podlesnyak A.A., Keller L., Prokeš K., Sikolenko V.V., Park J.-G., Skryabin Yu.N., Pirogov A.N., J. Magn. Magn. Mater., 2006, 300, e411; doi 10.1016/j.jmmm.2005.10.179

11. Bak P., Rep. Prog. Phys., 1982, 45, 587; doi 10.1088/0034-4885/45/6/001.

12. Huse D.A., Fisher M., Phys. Rev. B, 1984, 29, 239; doi 10.1103/PhysRevB.29.239

13. Den Nijs M., In: Phase Transitions and Critical Phenomena, Vol. 12, Domb C., Lebowitz J.L. (Eds.), Acedemic Press, London, 1988, p. 219-333.

14. Patrykiejew A., Sokołowski S., Binder K., Surface Sci., 2002, 512, 1; doi 10.1016/S0039-6028(02)01702-8

15. Frenkel Y.I., Kontorova T., Zh. Eksp. Theor. Fiz., 1938, 8, 1340 (in Russian).

16. Lomdahl P.S., Srolovitz D.J., Phys. Rev. Lett., 1986, 57, 2702; doi 10.1103/PhysRevLett.57.2702

17. Hamilton J.C., Phys. Rev. Lett., 2002, 88, 126101; doi 10.1103/PhysRevLett.88.126101

18. Daruka I., Hamilton J.C., J. Phys.: Condens. Matter, 2003, 15, 1827; doi 10.1088/0953-8984/15/12/302

19. Braun O.M., Kivshar J.S., Phys. Rep., 1998, 306, 1; doi 10.1016/S0370-1573(98)00029-5

20. Braiman Y., Baumgarten J., Jortner J., Klafter J., Phys. Rev. Lett., 1990, 65, 2398; doi 10.1103/PhysRevLett.65.2398

21. Braiman Y., Baumgarten J. Klafter J., Phys. Rev. B, 1993, 47, 11159; doi 10.1103/PhysRevB.47.11159

22. Vanossi A., Franchini A., Bortolani V., Surface Sci., 2002, 502-503, 437; doi 10.1016/S0039-6028(01)01990-2

23. Phillips J.M., Dash J.G., J. Stat. Phys., 2005, 120, 721; doi 10.1007/s10955-005-5252-x

24. Hartnett A.S., Phillips J.M., Phys. Rev. B, 2008, 77, 035408; doi $10.1103 /$ PhysRevB.77.035408

25. Patrykiejew A., Rżysko W., Sokołowski S., J. Phys. Chem. C, 2011, 116, 753; doi 10.1021/jp208323b

26. Markov I., Trayanov A., J. Phys.: Condens. Matter, 1990, 2, 6965; doi 10.1088/0953-8984/2/33/009

27. Houlrik J. M., Landau D.P., Phys. Rev. B, 1991, 44, 8962; doi $10.1103 /$ PhysRevB.44.8962

28. Diao J., Gall K., Dunn M.L., Nat. Mater., 2003, 2, 656; doi 10.1038/nmat977 
29. Chua Y., Leahy B., Zhang M., You S., Lee K.Y.C., Coppersmith S.N., Lin B., PNAS, 2013, 110, 824; doi 10.1073/pnas.1101630108

30. Patrykiejew A., Condens. Matter Phys., 2012, 15, 23601. doi 10.5488/CMP.15.23601

31. Patrykiejew A., Sokołowski S., J. Chem. Phys., 2012, 136, 144702; doi 10.1063/1.3699330.

32. Patrykiejew A., J. Phys.: Condens. Matter, 2013, 25, 015001; doi 10.1088/0953-8984/25/1/015001

\title{
Формування домен-стінки в бінарних наноскопічних скінченних системах
}

\begin{abstract}
А. ПатрикєєВ, С. Соколовскі
Відділ моделювання фізико-хімічних процесів, Університет Марії Кюрі-Склодовської, Люблін, Польща

Використовуючи просту одновимірну типову модель Френкеля-Конторової, ми показали, що скінченні співвимірні ланцюжки можуть зазнавати співвимірний-неспіввимірний (C-IC) перехід, якщо ланцюжок забруднений ізольованими домішками, дочепленими до кінців ланцюжка. За допомогою методу Монте Карло (МС) показано, що таке ж явище виникає в двовомірних системах з домішками, розміщеними на периферії скінчених співвимірних кластерів.
\end{abstract}

Ключові слова: бінарна суміш, співвимірні-неспіввимірні переходи, симуляції Монте Карло, скінченні системи, модель Френкеля-Конторової 
$\overline{ }$ 